\title{
Development and Static Mode Characterization of a New Low-Loss AC Switch Based on Super-gain BJT
}

\author{
Zheng Ren, Sébastien Jacques, Yubing Song, Adelphe Caldeira, Guillaume Goubard, Ambroise Schellmanns and \\ Nathalie Batut \\ GREMAN, CNRS-UMR 7347, University of Tours, Tours 37200, France
}

Received: July 11, 2013 / Accepted: September 06, 2013 / Published: February 28, 2014.

\begin{abstract}
This paper deals with an innovative low-loss AC switch, named as TBBS (transistor based bidirectional switch), based on the association of super-gain BJTs developed by the GREMAN laboratory. The main characterization results of the super-gain BJT are reminded to identify the key parameters that are essential to build the TBBS. A complete characterization database in static mode of this new AC switch is discussed. In particular, its forward and reverse-biased features have been measured to see the evolution of the DC current gain as a function of the current density. The TBBS makes sense when using the super-gain BJT (bipolar junction transistor) in reverse mode. It means that the reverse DC current gain has to be sufficient (at least higher than 1 compared with the conventional BJT one). This new AC switch is bidirectional in current and voltage, totally controllable (turn-on and turn-off) and the most attractive solution in terms of on-state power losses. Further, its manufacturing process is as easier as existing device such as triac.
\end{abstract}

Key words: AC switch, BJT, super-gain, static characterization, low power consumption.

\section{Introduction}

The efficient management of energy consumption is always a priority in the development of power electronics [1, 2]. Due to the constant rise of the fossil energy price and the greenhouse effect caused by the emission of $\mathrm{CO}_{2}$, more and more attention is drawn to solving this problem.

Recent studies have highlighted that the household appliances (fridges, lighting systems, air-conditioners, televisions, computers ...) represent a great part of the electrical energy consumption [3, 4]. For example, statistical studies show that $37 \%$ of the total electricity production in USA is consumed by domestic applications and 43\% in France [5].

The efficient management of electrical energy can be achieved by the application of intelligent grid and the realization of smart buildings [6]. The approach of

Corresponding author: Sébastien Jacques, researcher, research field: new power devices dedicated to energy conversion and energy efficiency. E-mail: sebastien.jacques@univ-tours.fr. these solutions requires the replacement of conventional mechanical and electromechanical switches embedded in walls by performing power electronic devices. In particular, it means that these devices must have low conduction power dissipation and be controllable in both on and off-states since they are connected to the AC mains. The achievement of this goal needs to propose innovative AC switch structures based on high performances (more efficient and more reliable) electronic components.

The GREMAN laboratory has recently developed a new super-gain BJT (bipolar junction transistor) following the requirements described previously [7]. Compared with standard BJT structures, several physical improvements have been made to obtain a super-gain (higher than 100) and low saturation voltage (lower than $0.3 \mathrm{~V}$ ) which leads to low consumption in on-state [8].

In the following of this paper, the physical structure and the main characterization results of the $5 \mathrm{~A}, 600 \mathrm{~V}$, super-gain BJT are reminded. The results are particularly compared with conventional marketed BJT 
to highlight the advantages of this new power device. Then, an innovative low-loss AC switch is proposed, based on the association of the basic super-gain BJT unit. A complete characterization database in static mode (forward and reverse-biased) is discussed. This new device points the way towards energy efficiency switch dedicated to domestic applications.

\section{Context Reminder and New AC Switch Proposal}

\subsection{State-of-the-Art on AC Switching Solutions}

After decades of development, there have existed several AC switch solutions on the market [9]. These solutions are listed in Table 1 with their own advantages (+) and drawbacks (-).

A comparison on on-state power dissipation, controllability (both turn-on and turn-off) and price is proposed respectively for each solution.

The use of two SCRs (silicon controlled rectifiers) connected in antiparallel enable low on-state power dissipation (about 1 W/A) because of the low voltage drop of each thyristor $(\approx 1 \mathrm{~V})$. It is also a very completive AC switch solution in terms of price. The main drawback of this AC switch is its controllability and particularly, during the turn-off. Indeed, thyristor conducts when its gate receives a current trigger and continues to conduct while it is forward biased. The turn-off of thyristor relies only on reversal current. That means the device cannot be turned-off using an external drive circuit which makes it difficult to apply for DC current. The same problem exists for triac (triode for alternating current) since this power component is based on the association in antiparallel of two SCRs. Diode bridge/IGBT and IGBTs/diodes are controllable in on and off-states, but the application of power diodes makes them less competitive in terms of conduction losses. The total voltage drop of its structure (Power diodes + controllable switch) can achieve $2 \mathrm{~V}$, which means the on-state power dissipation could reach 2 W/A. The use of SJ-MOSFET/Diodes to build AC switching is not suitable for power applications, because its price become unacceptable when the active area of SJ-MOSFET is expanded.

\subsection{Super-gain BJT Concept and Main Experimental Results Reminder}

The drawbacks of the existing AC switch solutions highlighted previously prompt the GREMAN laboratory to propose a new AC switch solution dedicated to efficient energy applications.

Before explaining the new AC switch structure, it is essential to remind the super-gain BJT structure and its main characterization results. The key features of the device are: typical breakdown voltage of $600 \mathrm{~V}$, current gain higher than 100 and low on-state power dissipation within $0.3 \mathrm{~W} / \mathrm{A}$.

Fig. 1 shows the physical structure of the device. The physical structure of the super-gain BJT is composed of two parts. The first one is a thin $(<2 \mu \mathrm{m})$ and weakly doped $\left(<10^{15} \mathrm{~cm}^{-3}\right)$ P-type base under the Emitter, used for conducting the collector-base current flow. The second part is composed of a thick $\mathrm{P}+$ well under the thin base, designed to reduce the

Table 1 Existing solutions for AC switching. Advantages (+) and drawbacks (-) are particularly highlighted.

\begin{tabular}{lll}
\hline Solution for AC switching & SCRs & Triac \\
\hline Diode bridge + IGBT
\end{tabular}




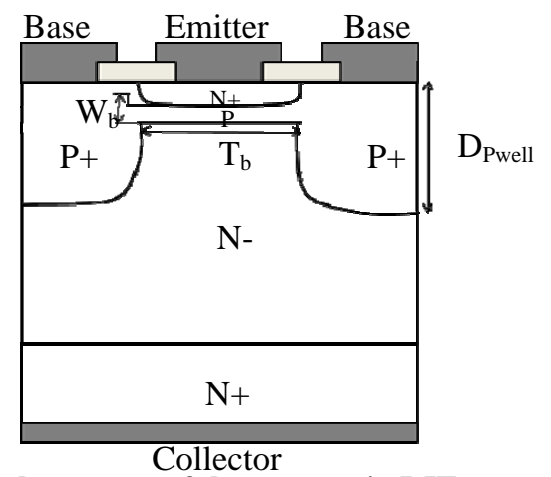

Fig. 1 Physical structure of the super-gain BJT.

specific resistance of base and predominantly to protect the thin base [10].

The geometric structure of the device has a significant influence on its electrical performances. Eq. (1) reminds the relation between the DC current gain $\left(h_{F E}\right)$ and the physical parameters of the device (thickness, doping concentrations, diffusion coefficients) [11].

$$
h_{F E}=\frac{W_{e} \cdot D_{n} \cdot N_{D e}}{W_{b} \cdot D_{p} \cdot N_{A b}}
$$

$h_{F E}$ : current gain;

$W_{b}$ : base thickness $(\mu \mathrm{m})$;

$W_{e}$ : emitter thickness $(\mu \mathrm{m})$;

$D_{n}$ : electron diffusion coefficient $\left(\mathrm{cm}^{2} \cdot \mathrm{s}^{-1}\right)$;

$D_{p}$ : hole diffusion coefficient $\left(\mathrm{cm}^{2} \cdot \mathrm{s}^{-1}\right)$;

$N_{A b}$ : base doping concentration $\left(\mathrm{cm}^{-3}\right)$;

$N_{D e}$ : emitter doping concentration $\left(\mathrm{cm}^{-3}\right)$.

According to the original design of the super-gain BJT, many CAD simulations have been performed to find the optimal value of all parameters presented in Eq. (1). The simulation results have particularly demonstrated that a depth value of $12 \mu \mathrm{m}$ for the $\mathrm{P}^{+}$ well $\left(D_{P \text { well }}\right)$ is enough to reach the targeted breakdown voltage (600 V). The base thickness of $1 \mu \mathrm{m}$ has been proven necessary to achieve the current gain higher than 100 [11]. The base width is fixed to $4 \mu \mathrm{m}$ with a doping concentration about $6 \times 10^{14} \mathrm{~cm}^{-3}$ to ensure the best compromise between the on-state power dissipation (lower than $0.3 \mathrm{~W} / \mathrm{A}$ ) and the off-state breakdown voltage (600 V).

All of the physical improvements give the super-gain
BJT new features in DC current gain, breakdown voltage and saturation voltage drop. First results of forward-biased characterization have already been introduced [12]. This paper describes the main characterization results in static mode as a function of junction temperature to complete the initial database presented in previous works. In particular, Fig. 2 shows the breakdown voltages $\left(V_{C E O}\right.$ and $\left.V_{C B O}\right)$ and the evolution of direct current gain $\left(h_{F E}\right)$ as a function of the collector current density $\left(J_{C}\right)$ at various levels of junction temperature (from $25^{\circ} \mathrm{C}$ to $125^{\circ} \mathrm{C}$ ).

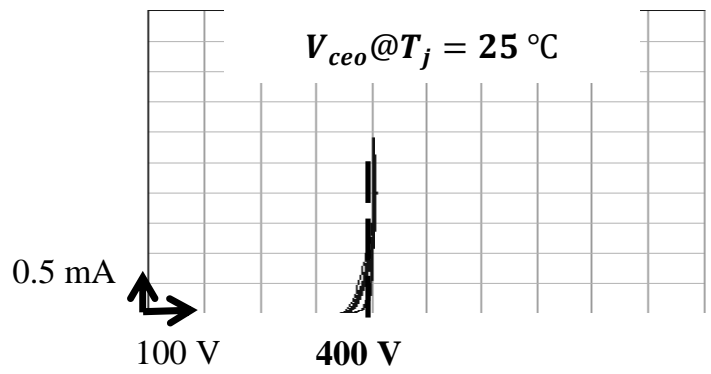

(a)

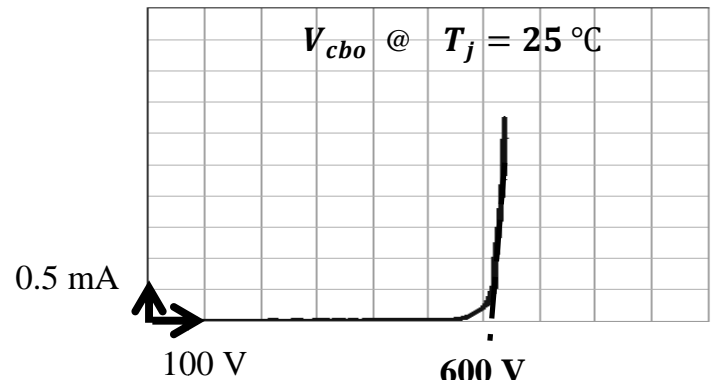

(b)

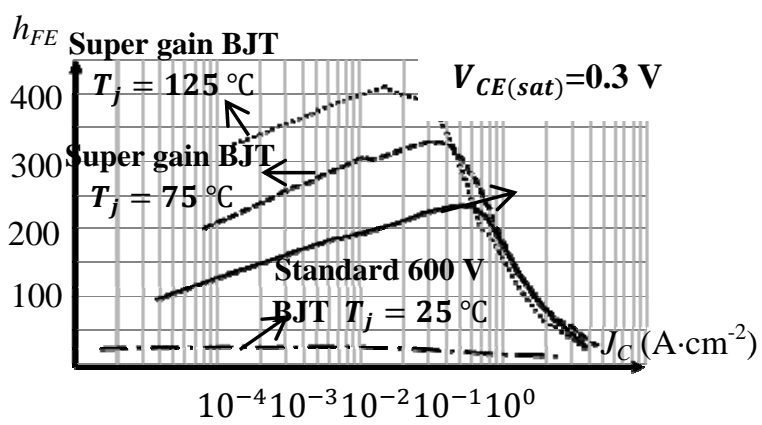

(c)

Fig. 2 Forward-biased characterization results of the super-gain BJT: (a) Collector-emitter breakdown voltage $\left(V_{C E O}\right)$ at $T_{j}=25{ }^{\circ} \mathrm{C}$; (b) collector-base breakdown voltage $\left(V_{C в о}\right)$ at $T_{j}=25{ }^{\circ} \mathrm{C}$; (c) forward-biased DC current gain $\left(h_{F E}\right)$ as a function of collector current density $\left(J_{C}\right)$ at 3 levels of junction temperature and $V_{C E \text { (sat) }}=0.3 \mathrm{~V}$. 
From the characterization results, the collector-emitter and collector-base breakdown voltages ( $V_{C E O}$ and $\left.V_{C B O}\right)$ are about $400 \mathrm{~V}$ and $620 \mathrm{~V}$ respectively. It is important to notice that the

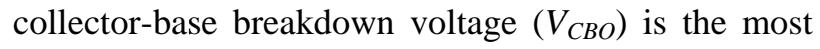
meaningful parameter to build a new AC switch. Moreover, it has to declare that the $\mathrm{V}_{\mathrm{CEO}}$-parameter (at least equal to $400 \mathrm{~V}$ ) is specifically optimized for the development of the new AC switch. However, the GREMAN laboratory is capable of fabricating $600 \mathrm{~V}$ super-gain BJT if this standalone device could be used in domestic applications.

Regarding the DC current gain, the gummel curve (Fig. 2c) shows a very encouraging performance since it can reach more than 200 at ambient temperature $\left(25^{\circ} \mathrm{C}\right.$ ) and more than 300 for junction temperatures from $75{ }^{\circ} \mathrm{C}$ to $125{ }^{\circ} \mathrm{C}$. A standard and marketed $5 \mathrm{~A}$, $600 \mathrm{~V}$ BJT (ST1802HI) with the same active area is also characterized for comparison. From its gummel curve presented in Fig. 2c, the $h_{F E}$-parameter of the super-gain BJT is about ten times higher than the conventional bipolar component (ST1802HI) at the same level of junction temperature. The reverse-biased DC current gain $\left(h_{F C}\right)$ is also very promising. The characterization result of this parameter will be discussed in the fourth section of this paper.

\subsection{New AC Switch Proposal}

The promising performances of the super-gain BJT give the GREMAN laboratory a possibility to propose an innovative AC switch structure, which is named as TBBS (transistor based bidirectional switch). The TBBS is achieved by associating two super-gain BJTs in anti-series, with their emitters connected and their bases in common. Fig. 3 shows the representative diagram of the TBBS.

The basic functionality of the TBBS is as follows: when one super-gain BJT is forward-biased, the other is reverse-biased. So, it means that each BJT must operate in forward and reverse modes since the switch is connected to the AC mains. This operation mode

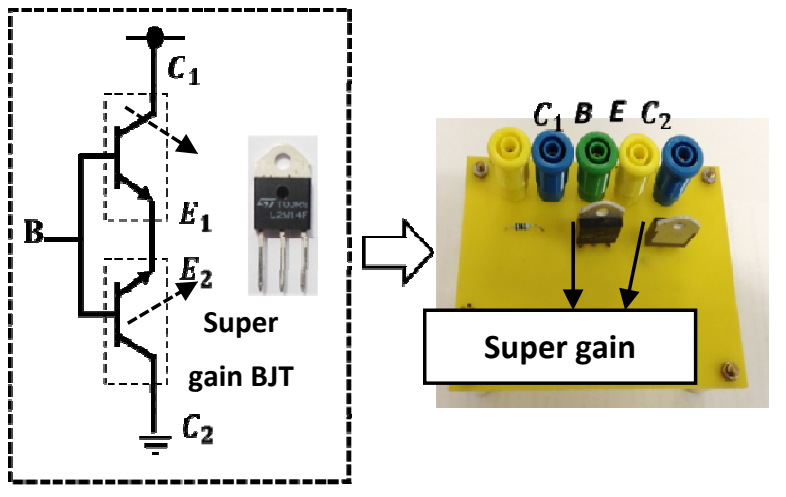

Fig. 3 Representative diagram of the TBBS.

requires that each basic BJT unit must have a sufficient reverse-biased gain (at least higher than 1 compared with conventional BJT) to conduct enough current. It is important to notice that the absolute maximum current of the TBBS is assured by the $h_{F C}$-parameter of a single super-gain BJT. This symmetrical structure is controllable in on and off-states and is bidirectional in current and voltage. More important, its power dissipation during the on-state is lower than the other existing solutions of AC switching (in particular, diode bridge/IGBT or MOSFETs/diodes that are penalized by the high conduction losses of the diodes) because of its low saturation voltage $V_{C E(s a t)}$.

The complete characterization database of the TBBS is presented in the next section of this paper.

\section{Experimental Procedure}

The aim of the experimental procedure is the static characterization of the TBBS in both forward and reverse modes, more specifically their forward-biased breakdown voltages and the performances of gain as a function of the collector current density at several levels of junction temperature. Further, the performance of the reverse-biased DC current gain $\left(h_{F C}\right)$ of the super-gain BJT basic unit is particularly highlighted because of its significant impact on the functionality of TBBS. At the end, the voltage drop of the TBBS at stranded test conditions (used by semiconductor device manufacturers) will be measured and compared with other AC switch solutions (e.g., 5 A, $600 \mathrm{~V}$ triac). 


\subsection{Test Bench Presentation and Precautions}

Fig. 4 shows the test bench used for the characterization procedure. It consists of a Tektronix 370 A curve tracer, a Haake F6 oil bath and a 3-pin socket. This characterization is carried out in a temperature-controlled environment in order to observe the thermal impacts on the electrical behavior of the TBBS. Polarization of components and acquisition of data were accomplished by the curve tracer, and the 3-pin socket is used to establish the connection between the oil bath and the curve tracer.

Several precautions should be taken during the experimental measurements. Regarding the oil bath, the maximum temperature measured must be lower than the boiling point of the dielectric oil to prevent the toxic vapor. A dielectric oil (3M Fluorinert FC40), whose boiling point equals to $165{ }^{\circ} \mathrm{C}$, is used. The maximum test temperature is limited to $125{ }^{\circ} \mathrm{C}$. The estimation of the collector current $\left(I_{C}\right)$ and $V_{C E \text { (sat) }}$ is indispensable to ensure that the device operates in the SOA (safety operating area). If the SOA is not specified in the datasheet, it is necessary to control the $I_{C}$ and $V_{C E(s a t)}$-parameters within the maximum power dissipation of the device. Finally, an exceedingly high temperature could harden the PVC cables in the dielectric oil, which could lead to a risk of short circuit. So, it is recommended to use the silicone cables which can withstand a higher measurement temperature.

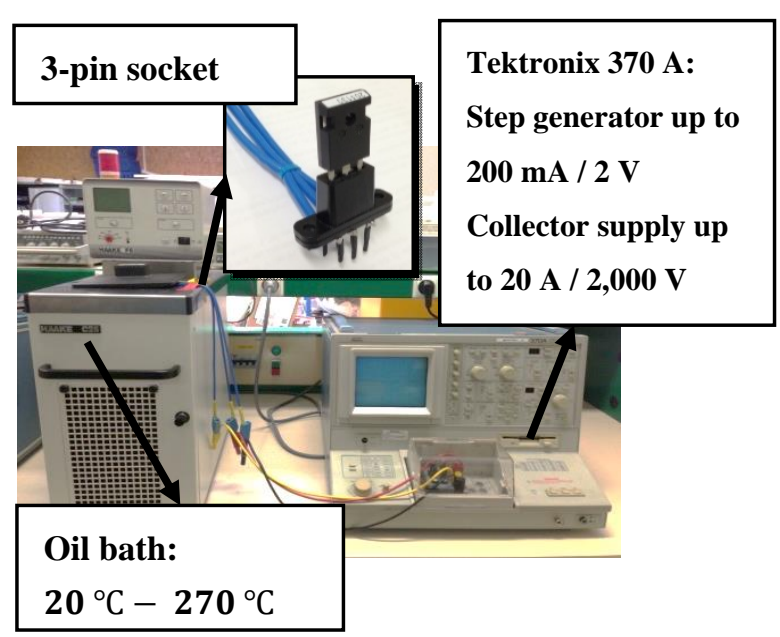

Fig. 4 Test bench used for the characterization procedure.

\subsection{Process of Experiment}

The characterization is carried out both in forward and reverse-bias at three levels of junction temperature $\left(25,75,125{ }^{\circ} \mathrm{C}\right)$. Regarding the forward-biased characterization, the studies on the breakdown voltage $\left(V_{\text {СЕО }}, V_{\text {Сво }}\right)$ and the evolution of the gain $\left(h_{F E}\right)$ as a function of the current density $\left(J_{C}\right)$ at various junction temperatures have been done for the super-gain BJT and the new TBBS respectively. To obtain the gummel plot, the base current $\left(I_{B}\right)$ of the component varied from $10 \mu \mathrm{A}$ to $100 \mathrm{~mA}$ and then, for each level of temperature measured, the couple of parameters $\left(h_{F E}, J_{C}\right)$ is calculated and plotted on the same graph. In this way, the evolution of the gain versus current density for different junction temperatures is obtained.

The same measurement procedure is applied for the reverse-biased characterization. The polarization of the device under test is inverted by exchanging the pins of collector and emitter, in the meantime keeping the same polarization of base as previous.

The measurement of the on-state saturation voltage of TBBS is carried out with a driving current $I_{B}=500$ $\mathrm{mA}$ and at the typical test conditions $\left(T_{j}=25{ }^{\circ} \mathrm{C}, I_{C}=1\right.$ A) defined by semiconductor device manufacturers.

\section{Experimental Results and Discussion}

\subsection{Breakdown Voltage}

Fig. 5 shows the forward-biased breakdown voltage of the proposed AC switch TBBS at the operational temperature of $25{ }^{\circ} \mathrm{C}$. This breakdown voltage value reaches typically $620 \mathrm{~V}$.

It is important to notice that this value remains constant despite the change of the junction temperature (from $25{ }^{\circ} \mathrm{C}$ up to $125{ }^{\circ} \mathrm{C}$ ). This phenomenon is also observed for the other breakdown voltages, both in forward-biased $\left(V_{C B O}\right)$ and in reverse-biased $\left(V_{E C O}\right)$. In addition, their shapes are almost the same except their values. All the measurement values of the forward-biased and reverse-biased breakdown voltage $\left(V_{C E O}, V_{C B O}, V_{E C O}\right)$ are summed up in Table 2. 


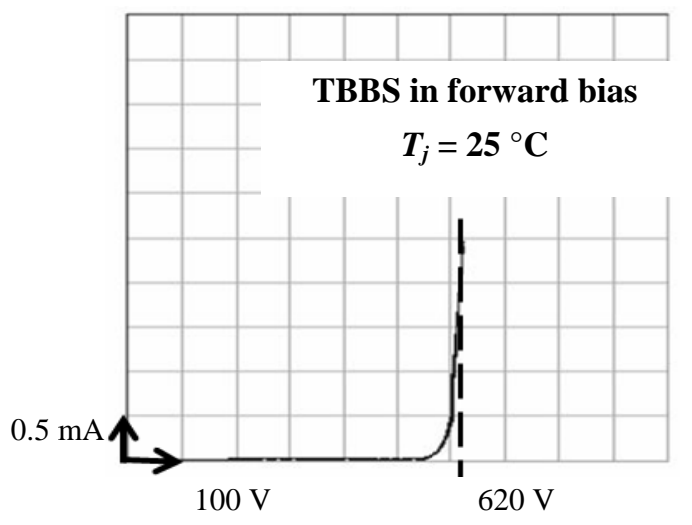

Fig. 5 Breakdown voltage of the TBBS at $T_{j}=25^{\circ} \mathrm{C}$.

Table 2 Forward-biased and reverse-biased breakdown voltages of the super-gain BJT (average value of 5 tested super-gain BJTs) and the TBBS.

\begin{tabular}{llll}
\hline & & Super-gain BJT & TBBS \\
\hline \multirow{2}{*}{ Forward-biased } & $V_{C E O}$ & $400 \mathrm{~V}$ & $620 \mathrm{~V}$ \\
& $V_{C B O}$ & $620 \mathrm{~V}$ & $620 \mathrm{~V}$ \\
\hline Reverse-biased & $V_{E C O}$ & $6 \mathrm{~V}$ & $620 \mathrm{~V}$ \\
\hline
\end{tabular}

The reverse-biased breakdown voltage of bipolar components is observed much lower than that of forward-biased. This is because the emitter layer is much thinner and much more doped than the collector layer.

Regarding the TBBS, the forward and reverse breakdown voltages are the same because of its symmetrical structure. More specifically, the two base-emitter junctions of the super-gain BJT basic units are all short-circuited by the common base of TBBS, making the total breakdown voltage of this bidirectional structure equals to the $V_{C B O}$ of a single super-gain BJT $(620 \mathrm{~V})$. The identical voltage of TBBS in both forward and reverse direction makes it suitable for AC voltage applications, which is an essential feature for AC switching.

\subsection{Current Gain in Both Forward and Reverse-Biased Mode}

Compared with the breakdown voltage $V_{C E O}$ and $V_{C B O}$ of the bipolar components which represent their capacities of supporting the reverse voltage, the gain, $h_{F E}$ at forward-biased or $h_{F C}$ at reverse-biased, shows the maximum capacity of conducting the current flows.
These two parameters are critical for the design and choose of a switch. Previously, the study on the breakdown voltages has been introduced, now the study on the gain will be presented.

The gummel curve exhibits the evolution of the DC current gain versus the current density. The active area of the super-gain BJT and TBBS are both $25 \mathrm{~mm}^{2}$. They have the same active area because the two super-gain BJTs which compose the TBBS are associated in anti-series, the current flow passes through each super-gain BJT successively. This is contrary of the active area relation between thyristor and TRIAC (anti-parallel). It should also be noticed that the $V_{C E(s a t)}$ of each component is fixed to $0.3 \mathrm{~V}$ and the junction temperature $T_{J}$ fixed at $25{ }^{\circ} \mathrm{C}$ in order to make sure that their curves are comparable.

Fig. 6 shows the reverse-biased DC current gain $\left(h_{F C}\right)$ as a function of the emitter current density $\left(J_{E}\right)$ for the two studied components. Regarding the $h_{F C}$-parameter, the super-gain BJT has an encouraging performance. It means that the device can conduct a significant current flow even if it is reverse-biased. This is the most important feature of super-gain BJT making it suitable to build the TBBS. The $h_{F C}$-parameter value of TBBS is observed the half of the super-gain BJT one for the same level of collector current density. The reason is that the driving current of TBBS is divided into the two super-gain BJTs. So, it means that the reverse-biased super-gain BJT of TBBS can only receive half of the $I_{B}$ regarding to the single super-gain $B J T$, so the generated $I_{C}$ becomes the half compared with a single

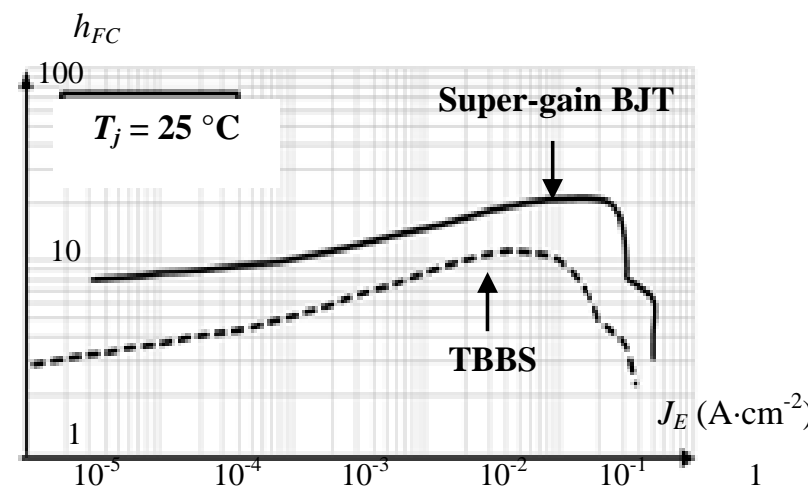

Fig. $6 \mathrm{DC}$ current gain in reverse mode at $T_{j}=25{ }^{\circ} \mathrm{C}$ and $V_{C E(s a t)}=0.3 \mathrm{~V}$. Comparison super-gain BJT vs. TBBS. 
super-gain BJT. Taking their identical curve forms, this experimental figure proves us that the performance of TBBS is defined by that of the reverse-biased super-gain BJT.

Fig. 7 shows a comparison of the Gummel curve in forward bias between the super-gain BJT and the TBBS. The experimental results exhibit that the DC current gain (in direct mode, $h_{F E}$ ) of the super-gain BJT is much higher than any other marketed bipolar transistor since it can reach more than 200 and this value goes even higher when the junction temperature increases. This feature suits its original design concept. The thinner base gives a higher gain to super-gain BJT. This also means that the base current $\left(I_{B}\right)$ can be smaller for the same level of collector current $\left(I_{C}\right)$, which reduces the consumption of the control circuit.

The forward-biased gain of TBBS is determined by the reverse-biased gain of a single super-gain BJT. Its maximum value is equal to 10 (Fig. 6), which is great enough to be the switch for household appliances. This value also exhibits that the TBBS is capable of passing through the alternative current flow because of its symmetrical structure, which is another essential feature for an AC switch.

\subsection{On-State Power Dissipation}

The conduction power losses are also a critical parameter in practice to choose a power device. Regarding bipolar transistors, the on-state power losses are generally limited by the saturation voltage $\left(V_{C E(s a t)}\right)$.

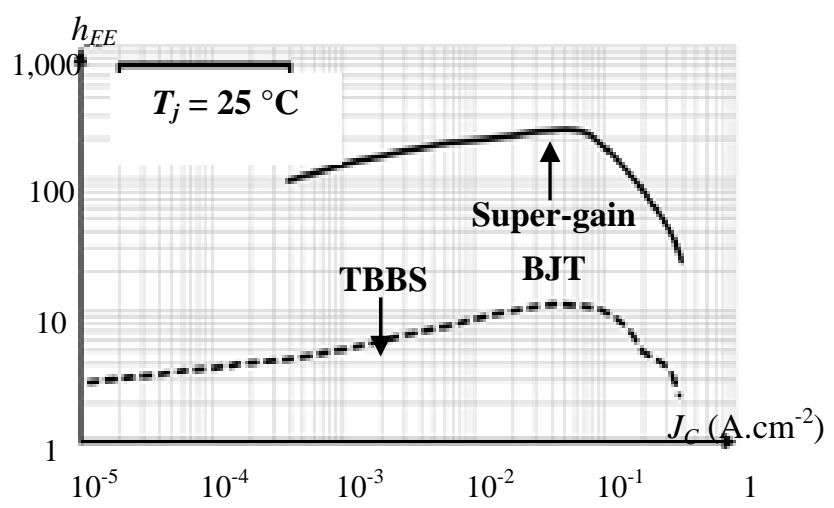

Fig. $7 h_{F E}\left(J_{C}\right)$ at $T_{j}=25^{\circ} \mathrm{C}$ and $V_{C E(s a t)}=0.3 \mathrm{~V}$.
For instance, the $V_{C E(s a t)}$-parameter of the super-gain BJT and the TBBS has been measured at the typical conditions generally defined by the device manufacturer. For a collector current of $1 \mathrm{~A}$ and a junction temperature of $25^{\circ} \mathrm{C}$, the $V_{C E \text { (sat) }}$ of super-gain BJT and TBBS equal $0.20 \mathrm{~V}$ and $0.29 \mathrm{~V}$ respectively, which means on-state power losses of $0.20 \mathrm{~W} / \mathrm{A}$ and 0.29 W/A.

For an AC switch, this level of on-state power dissipation (0.29 W/A) is amazing. To highlight this feature of the TBBS, a marketed and widely used $5 \mathrm{~A}$, $600 \mathrm{~V}$ triac has been characterized to compare its performances with the TBBS ones. The I-V curves of each component are presented in Fig. 8. It is important to notice that the measurements have been performed at the same junction temperature $\left(25^{\circ} \mathrm{C}\right)$ and the same driving current (500 mA). For the same level of current flow (1 A), the voltage drops of TBBS and Triac are about $0.29 \mathrm{~V}$ and $1 \mathrm{~V}$, respectively. It means that their on-state power dissipations are equal to $0.29 \mathrm{~W} / \mathrm{A}$ and 1 W/A, respectively. This result presents a great advantage of TBBS in terms of power dissipation compared with triac.

Table 3 gives the comparison on the power dissipation of several AC switch solutions used in power electronics. The results clearly show that the TBBS exhibits the most attractive performances in terms of on-state power losses. The application of this kind of device can not only save energy consumptions,

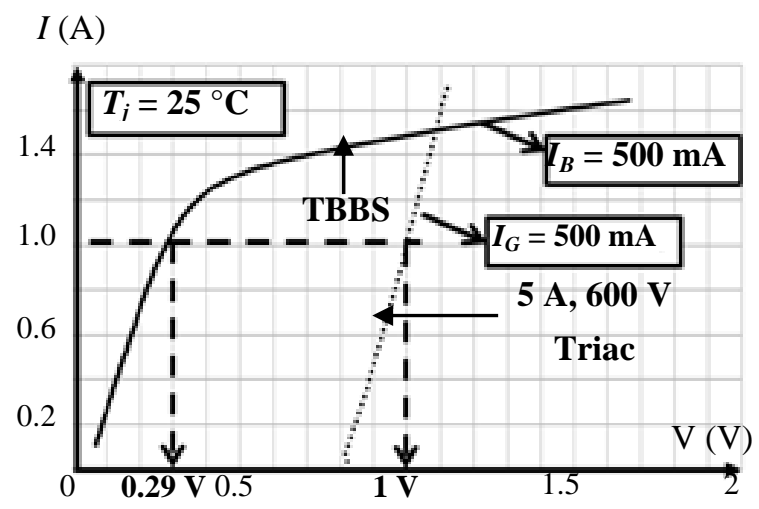

Fig. 8 On-state voltage drop. Comparison TBBS vs. 5 A, 600 V Triac. 
Table 3 On-state power dissipation, comparison of existing AC switching solutions.

\begin{tabular}{ll}
\hline AC switch solutions & $\begin{array}{l}\text { On-state power dissipation } \\
\left(I c=1 \mathrm{~A}, T_{i}=25{ }^{\circ} \mathrm{C}\right)\end{array}$ \\
\hline Thyristor in antiparallel & $1 \mathrm{~W} / \mathrm{A}$ \\
TRIAC & $1 \mathrm{~W} / \mathrm{A}$ \\
Diode bridge + IGBT & $2 \mathrm{~W} / \mathrm{A}$ \\
IGBTs + Diodes & $2 \mathrm{~W} / \mathrm{A}$ \\
SJ-MOSFET + Diodes & $0.9 \mathrm{~W} / \mathrm{A}$ \\
TBBS & $0.29 \mathrm{~W} / \mathrm{A}$ \\
\hline
\end{tabular}

but also reduce the heat at the same level of current flow thus minimize the size of heat-sink and extend the lifetime and reliability of the component.

\section{Conclusions}

The efficient management of domestic electrical energy imposes the development of high performance AC switch. To achieve this goal, the GREMAN laboratory has recently developed a new super-gain BJT. Its promising electrical performances on the reverse-biased DC current gain ( $h_{F C}$ higher than 10) and saturation voltage drop ( $V_{C E(s a t)}$ lower than $0.25 \mathrm{~V}$ ) give birth to a new structure of AC switch solution, named as TBBS (transistor based bidirectional switch). This innovative AC switch structure is characterized and is proven to be bidirectional in voltage and current and totally controllable (turn-on and turn-off). Its breakdown voltage is about $620 \mathrm{~V}$. This value is suitable for typical domestic applications. The maximum DC current gain of this AC switch is about 10 , which means that the device is capable of conducting a high current flow and easy to drive (smaller Base current). Its on-state voltage drop of 0.29 $\mathrm{V}$ makes it very competitive in the on-state power dissipation (0.29 W/A) compared with existing AC switch solutions (for example, triac).

The requirements of "smart buildings" and the domestic applications (lighting systems, computers, remote-control systems,etc.) impose the further study of this new AC switch solution focusing on the dynamic mode characterization and the development of the specific driving circuit. The performance of the super-gain BJT could also be improved to expand the scope of application of TBBS.

\section{References}

[1] Y.H. Lu, G.D. Micheli, Comparing system level power management policies, IEEE Design and Test of Computers 18 (2) (2001) 10-19.

[2] A. Amerasekera, Ultra low power electronics in the next decade, in: Proceedings of the IEEE International Symposium on Low-Power Electronics and Design, Austin, TX, USA, 2010, p. 237.

[3] G. Anastasi, F. Corucci, F. Marcelloni, An intelligent system for electrical energy management in buildings, in: Proceedings of the International Conference on Intelligent Systems Design and Applications, Cordoba, Argentina, 2011, pp. 702-707.

[4] P. Du, N. Lu, Appliance commitment for household load scheduling, IEEE Transactions on Smart Grid 2 (2) (2011) 411-419.

[5] Energy consumption by Sector and Source, US Energy Information Administration Web site, Washington DC 20585, July 10, 2013, http://www.eia.gov.

[6] B. Morvaj, L. Lugaric, S. Krajcar, Demonstrating smart buildings and smart grid features in a smart energy city, in: Proceedings of the International Youth Conference on Energetics, Leiria, 2011, pp. 1-8.

[7] L.V. Phung, C. Benboujema, N. Batut, J.B. Quoirin, A. Schellmanns, L. Jaouen, et al., Modeling of a new SOI bidirectional bipolar junction transistor for low-loss household appliances, IEEE Transactions on Electron Devices 58 (4) (2011) 1164-1169.

[8] L. Théolier, L.V. Phung, N. Batut, A. Schellmanns, Y. Raingeaud, J.B. Quoirin, BJT static behavior improvement by modification of the epitaxial layer, in: Proceedings of the International Conference on Microelectronics, Nis, 2010, pp. 79-82.

[9] L.V. Phung, N. Batut, A. Schellmanns, S. Jacques, A review on selected patents about trends regarding silicon monolithic power AC switches, Recent Patents on Electrical and Electronic Engineering 5 (3) (2012) 222-230.

[10] J.B. Quoirin, L.V. Phung, N. Batut, Bidirectional power switch controllable to be turned on and off, US Patent 20110121407 A1 (2010).

[11] L. Théolier, C. Benboujema, A. Schellmanns, N. Batut, Y. Raingeaud, J.B. Quoirin, BJT Application Expansion by Insertion of Super Junction, in: Proceedings of the International Symposium on Power Semiconductor devices and ICs, Hiroshima, Japan, 2010, pp. 157-160.

[12] C. Benboujema, S. Jacques, A. Schellmanns, N. Batut, J.B. Quoirin, L. Jaouen, et al., Characterization of a high gain BJT used in power conversion on AC mains, in: Proceedings of the IEEE Energy Conversion Congress and Exposition, Atlanta, USA, 2010, pp. 357-361. 